\title{
Treatability of different components of aphasia-Insights from a case study
}

\author{
Diane L. Kendall, PhD; ${ }^{1-2 *}$ Stephen E. Nadeau, MD $;^{2-4}$ Tim Conway, PhD; ${ }^{1,5}$ Renee H. Fuller, MA, \\ CCC-SLP; ${ }^{6}$ Alonso Riestra, MD; ${ }^{7}$ Leslie J. Gonzalez Rothi, PhD ${ }^{1-2,4}$ \\ ${ }^{1}$ Department of Veterans Affairs (VA) Rehabilitation Research and Development Brain Rehabilitation Research Center, \\ Malcom Randall VA Medical Center (VAMC), Gainesville, FL; ${ }^{2}$ Department of Neurology, University of Florida, \\ Gainesville, FL; ${ }^{3}$ McKnight Brain Institute, University of Florida, Gainesville, FL; ${ }^{4}$ Geriatric Research, Education, \\ and Clinical Center, Malcom Randall VAMC, Gainesville, FL; ${ }^{5}$ Department of Clinical and Health Psychology, Uni- \\ versity of Florida, Gainesville, FL; ${ }^{6}$ Cardinal Hill Rehabilitation Hospital, Lexington, KY; ${ }^{7}$ Department of Neurology, \\ Instituto Mexicano de Neurociencias (Mexican Institute of Neurosciences), Huixquilucan, México
}

\begin{abstract}
In this phase I clinical rehabilitation study, we investigated the effects of phonological rehabilitation for alexia and aphasia in an individual 54 years after a left-hemisphere ischemic infarction. In the context of a single-subject design, we studied whether treatment would improve phonological processing, reading, and generalization to untreated behaviors. While results showed a lack of generalization to real-word reading aloud, improvement was present in phonological processing, language function (Western Aphasia Battery Aphasia Quotient, Boston Naming Test, Reading Comprehension Battery for Aphasia), and auditory processing (Revised Token Test). Improvement in the lexical-semantic system was attributed to informal forced-use language treatment. We concluded that phonological therapies are unlikely to be successful unless a minimum initial level of phonological sequence knowledge exists; therapies that pressure subjects to use verbal communication can achieve clinically important gains in communicative ability that generalize to untreated behaviors. This study also demonstrates the importance of a careful analysis of the patient's language ability before a therapeutic strategy is chosen.
\end{abstract}

Key words: alexia, aphasia, chronic, constraint-induced language therapy, language function, nonword reading aloud, phonological processing, real-word reading aloud, rehabilitation, stroke, verbal communication.

\section{INTRODUCTION}

With the steady advance of the science of aphasia rehabilitation, the therapist now has several treatment options for any given patient. Treatment choice may hinge on the deficit that is most problematic to the patient. However, that deficit may not respond well to treatment. In this article, based on experience with two treatments provided to a single patient-one planned and

\footnotetext{
Abbreviations: $\mathrm{BNT}=$ Boston Naming Test, $\mathrm{C}=$ consonant, CILT $=$ constraint-induced language therapy, CTOPP $=$ Comprehensive Test of Phonological Processing, ITSACORR = independent time series analysis of autocorrelated data, LAC = Lindamood Auditory Conceptualization, LiPS = Lindamood Phoneme Sequencing, PDP = parallel distributed processing, RCBA = Reading Comprehension Battery for Aphasia, RR\&D = Rehabilitation Research and Development, RTT = Revised Token Test, V = vowel, VA = Department of Veterans Affairs, WAB = Western Aphasia Battery, WRMT-R = Woodcock Reading Mastery Test-Revised.

* Address all correspondence to Diane L. Kendall, PhD; VA RR\&D Brain Rehabilitation Research Center, Malcom Randall VAMC, Box 151A, 1601 SW Archer Road, Gainesville, FL 32608-1197; 352-376-1611, ext 5238; fax: 352-3792332. Email: kendadl@neurology.ufl.edu

DOI: 10.1682/JRRD.2005.01.0014
} 
less successful, one unplanned and more successful-we seek to elucidate the general problem of determining treatability before treatment is actually initiated.

We studied a right-handed 77-year-old woman with nonfluent, global aphasia and severe alexia 54 years after she experienced a massive left-hemisphere ischemic infarction. Motivated by results of developmental phonological dyslexia treatment in children [1-4], the success of acquired phonological alexia treatment in adults [5-10], and our own prior success with alexia treatment in an adult patient with stroke using a modified version of the Lindamood Phoneme Sequencing (LiPS) program [11-13], we provided the subject with 74 hours of phonological treatment. Most of the sessions were conducted in the subject's home. Before and after formal therapy, the therapist engaged the subject in extensive conversations about daily events and family history that was, in effect, an informal version of forced-use language therapy [14-15]. We present the details of these therapies and her responses and consider possible reasons for her response pattern.

\section{METHODS}

\section{Subject Description}

The subject was a 77-year-old right-handed female with 12 years of education who suffered a lefthemisphere ischemic infarction during the birth of her only son in 1945 (Figure 1) and was diagnosed with global aphasia immediately postonset. The subject reported that she went to an inpatient rehabilitation unit for 2 weeks immediately poststroke and received speechlanguage therapy. She was 54 years postonset at the time of this study, and her language indicated nonfluent aphasia with alexia, agraphia, and apraxia of speech. The subject provided her informed consent (University of Florida, form 545-99) prior to testing and treatment. Extensive speech, language, cognitive, and reading evaluations were conducted at the time of this study (Table). The subject currently lives with her husband in a retirement community and is independent in all activities of daily living except driving, reading, and writing. Her communication partners were limited to her husband and to friends with whom she interacted about once a week.

Her scores on standard measures of language function are recorded in the Table. Discourse performance on the Western Aphasia Battery (WAB) picture description was nonfluent and characterized by single-word and simple-phrase production (e.g., a house, picnic, a dog, lit- tle boy in the water). While impaired, her phonological sequence knowledge was evidenced by her ability to repeat some nonwords (Comprehensive Test of Phonological Processing [CTOPP]) [16]; with phonemic cues, she showed improvement in reading nonwords and confrontation naming.

In terms of silent reading abilities, unstandardized testing showed that the subject was able to identify real words (e.g., "point to the word ___") in an array of real and nonwords with 57 percent accuracy, match real words to appropriate pictures with 70 percent accuracy, match sentences to pictures with 70 percent accuracy, and match pseudo-homophonic words to pictures with 28 percent accuracy. The subject was able to read real words aloud with 47 percent accuracy and nonwords aloud with 0 percent accuracy. The disparity between the subject's poor performance on nonword and pseudohomophone reading and her performance on real-word reading suggests that she was reading by a whole-word route. Tests of phonological processing revealed impairment in her ability to discriminate, blend, and segment phonemes.

\section{Procedures}

\section{Experimental Design}

We used a multiple-baseline single-subject design in this study to evaluate the effects of a phonologically based intervention program. The subject initially received a series of diagnostic tests (language, speech, and reading). Stable baselines were established for treatment (nonword reading aloud), generalization (real-word reading aloud), and control (30-item Boston Naming Test [BNT]) [17] probes, which were administered prior to treatment initiation and weekly during treatment (Figure 2). The treatment and generalization probe stimuli were randomized each week to prevent potential order effects. One week after treatment termination, treatment, generalization, and control probes and posttest language measures were administered. A therapist uninvolved in the study administered the pretests and the treating therapist administered the posttests.

\section{Treatment Probes and Outcome Measures}

To determine whether phonological treatment improved phonological processing and the ability to read nonwords aloud, we administered treatment probes of consonant (C)-vowel (V) and CVC nonword reading aloud (e.g., "og," “zo," “dak”) and two phonological 


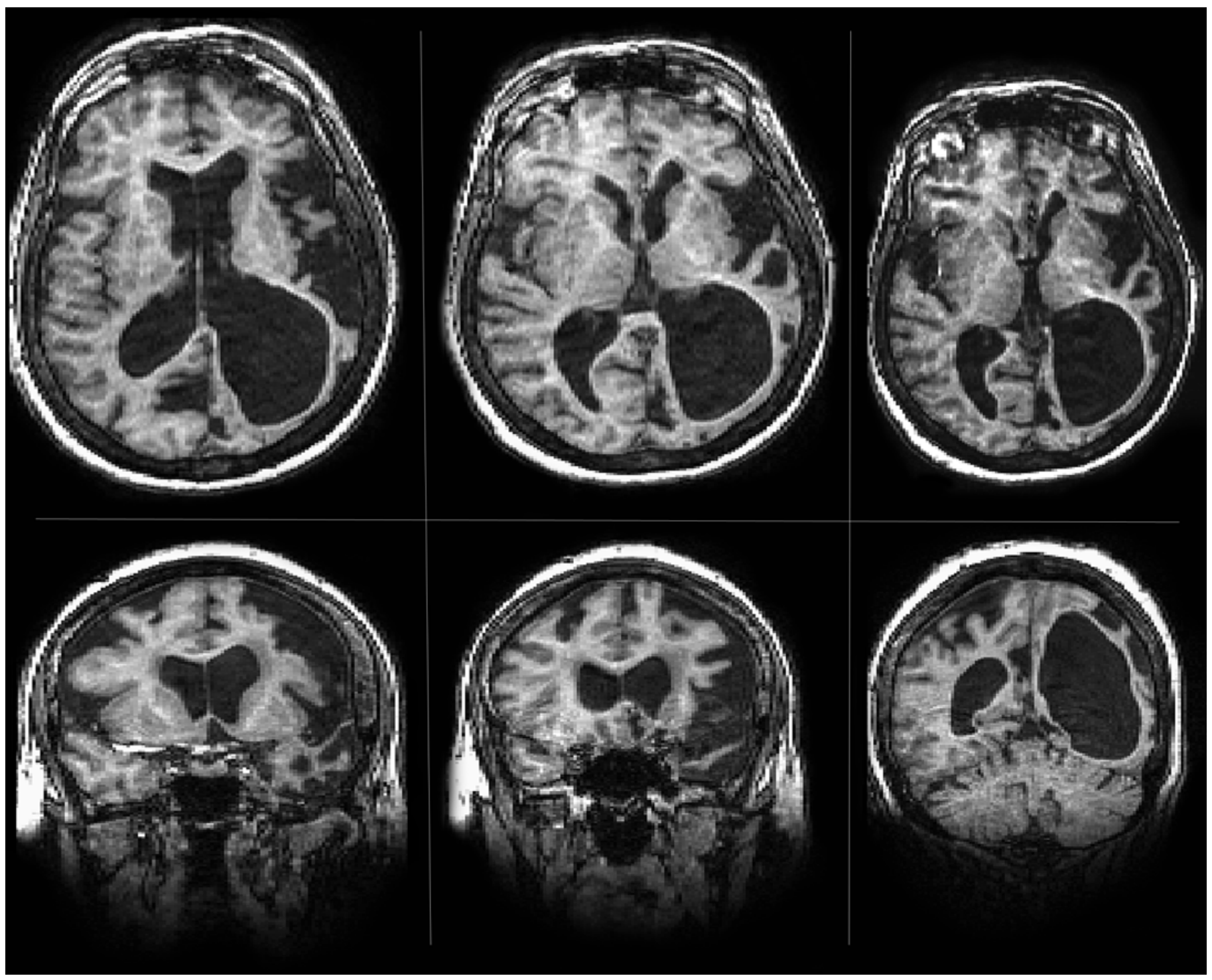

Figure 1.

Magnetic resonance images of 77-year-old female subject with left-hemisphere infarction at time of treatment (fluid attenuated inversion recovery sequence).

processing tests prior to treatment: the CTOPP [16], primarily a test of phonological production, and the Lindamood Auditory Conceptualization (LAC) [18], primarily a test of phonological perception.

To determine whether treatment generalized to untreated behaviors, we administered a real-word reading aloud generalization probe of single-syllable real words (e.g., man, room, week) as well as pre- and posttest measures of overall language function (WAB) [19], auditory processing (Revised Token Test [RTT]) [20], and reading (Reading Comprehension Battery for Aphasia [RCBA]) [21].
To determine whether treatment and generalization effects were maintained following treatment termination, we administered treatment, generalization, and control (BNT) probes 1 week after treatment termination.

\section{Treatment Description}

The rehabilitation program was a modified version of the LiPS program [11]. We administered phonological treatment 3-4 days/week, 1-2 hours/day for a total of 74 hours of therapy over 6 months. Treatment was administered at the Department of Veterans Affairs (VA) Rehabilitation Research and Development (RR\&D) Brain 
JRRD, Volume 43, Number 3, 2006

Table.

Summary of pre- and posttreatment standardized test results of 77-year-old female subject.

\begin{tabular}{|c|c|c|c|}
\hline Standardized Test & Pretreatment & Posttreatment & Total Possible \\
\hline \multicolumn{4}{|l|}{ Western Aphasia Battery [1] } \\
\hline Aphasia Quotient & 56.2 & 86.5 & 100 \\
\hline Information Content & 8 & 9 & 10 \\
\hline Fluency & 2 & 9 & 10 \\
\hline Yes/No Questions & 60 & 60 & 60 \\
\hline Auditory Word Recognition & 46 & 57 & 60 \\
\hline Sequential Commands & 39 & 72 & 80 \\
\hline Repetition & 37 & 76 & 100 \\
\hline Object Naming & 47 & 56 & 60 \\
\hline Word Fluency & 9 & 6 & 20 \\
\hline Sentence Completion & 8 & 10 & 10 \\
\hline Response Speech & 10 & 10 & 10 \\
\hline Revised Token Test $[2]$ & 8.817 & 10.779 & 15 \\
\hline Reading Comprehension Battery for Aphasia* [3] & 31 & 48 & - \\
\hline \multicolumn{4}{|l|}{ Comprehensive Test of Phonological Processing ${ }^{\dagger}$ [4] } \\
\hline Elision & 1 & 1 & - \\
\hline Blending Words & 2 & 4 & - \\
\hline Memory for Digits & 1 & 1 & - \\
\hline Nonword Repetition & 2 & 4 & - \\
\hline Rapid Digit Naming & 1 & 0 & - \\
\hline Rapid Color Naming & 1 & 1 & - \\
\hline Phoneme Reversal & 2 & 2 & - \\
\hline Rapid Object Naming & 1 & 1 & - \\
\hline Blending Nonwords & 2 & 4 & - \\
\hline \multicolumn{4}{|l|}{ Test of Adolescent and Adult Language ${ }^{\ddagger}$ [5] } \\
\hline Listening/Vocabulary & 3 & 8 & - \\
\hline Listening/Grammar & 3 & 3 & - \\
\hline Speaking/Vocabulary & 0 & 1 & - \\
\hline \multicolumn{4}{|l|}{ 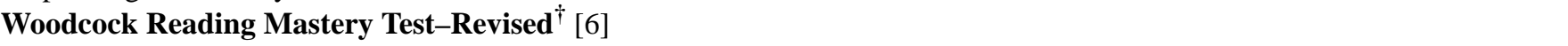 } \\
\hline Word Attack & 0 & 38 & - \\
\hline Word Identification & 10 & 10 & - \\
\hline \multicolumn{4}{|l|}{ Lindamood Auditory Conceptualization [7] } \\
\hline Total Converted Score & 27 & $58^{\S}$ & 100 \\
\hline
\end{tabular}

1. Kertesz A. The Western Aphasia Battery. Austin (TX): PRO-ED Inc; 1982.

2. McNeil MM, Prescott TE. Revised Token Test. Austin (TX): PRO-ED Inc; 1978.

3. LaPointe LL, Horner J. Reading Comprehension Battery for Aphasia. Austin (TX): PRO-ED Inc; 1979.

4. Wagner R, Torgesen J, Rashotte C. The Comprehensive Test of Phonological Processing. Austin (TX): PRO-ED Inc; 1998.

5. Hammill D, Brown V, Larsen S, Wiederholt JL. Test of Adolescent and Adult Language. Austin (TX): PRO-ED Inc; 1994.

6. Woodcock RW. Woodcock Reading Mastery Test-Revised. Circle Pines (MN): American Guidance Service, Inc; 1987.

7. Lindamood PC, Lindamood PD. Lindamood Auditory Conceptualization Test. Austin (TX): PRO-ED Inc; 1979.

*Raw score: subtests I-VI.

${ }^{\dagger}$ Standard score.

¥ Scaled score.

$\S_{\text {}}$ Test was not completed to ceiling cutoff criteria. Score may be underestimate.

Rehabilitation Research Center 1 day/week and in the home 2 to 3 days/week. We calculated that the unplanned treatment occurred for approximately 30 hours over the 6-month treatment period.
The LiPS program is arranged hierarchically and divided into three stages: (1) oral awareness training, (2) simple nonword training (e.g., single syllables), and (3) complex nonword training (2-3 syllables) [13]; 


\begin{tabular}{|c|c|}
\hline Real Words & Nonwords \\
\hline $\operatorname{man}$ & $a b$ \\
\hline time & op \\
\hline year & zo \\
\hline way & ka \\
\hline life & $\mathrm{a}$ \\
\hline hand & kep \\
\hline house & dak \\
\hline child & uk \\
\hline part & ta \\
\hline word & 1 \\
\hline fact & gar \\
\hline eye & es \\
\hline group & ve \\
\hline case & $\mathrm{u}$ \\
\hline war & fop \\
\hline line & ut \\
\hline side & gu \\
\hline night & o \\
\hline head & tas \\
\hline form & ov \\
\hline room & ba \\
\hline week & bap \\
\hline end & ig \\
\hline boy & de \\
\hline girl & hig \\
\hline car & ep \\
\hline use & sa \\
\hline law & lep \\
\hline \multirow[t]{7}{*}{ face } & og \\
\hline & ko \\
\hline & mub \\
\hline & ef \\
\hline & ta \\
\hline & nep \\
\hline & ir \\
\hline
\end{tabular}

Figure 2.

Real and nonword repeated generalization probe stimuli.

however, the subject was only able to complete the first two stages in the 74 hours. The patient was unable to progress to complex nonword training because of a significant speech-motor programming impairment. We taught the first stage, awareness of how the articulators move to produce phonemes, using Socratic questioning, which involved providing visual feedback of the subject's mouth from a mirror, verbal labels of the articulators' movements, voicing characteristics, and line drawings of the articulators. This multisensory approach provides the subject with in-depth perception (visual, auditory, and oral tactile-kinesthetic) of the individual phonemes. For example, the subject was asked to look at her mouth in the mirror, produce the phoneme $/ \mathrm{p} /$, and decide whether her lips or tongue made the primary movement to produce that particular phoneme. She was asked to decide if her lips popped apart quickly or if they moved slowly apart. Next, she was asked to place her fingers on her throat to decide if the phoneme $/ \mathrm{p} /$ was unvoiced (quiet) or voiced (noisy). Following the response, the phoneme $/ \mathrm{p} /$ was given the verbal label of a "quiet lip-popper." Next, the subject was asked to think of another sound that required the same lip-popping action of her mouth, but was voiced. The cognate phoneme /b/ was discovered and the verbal label "noisy lippopper" was applied. Finally, the subject was shown three different pictures and asked which picture best represented how her mouth moved to produce the "lippopper sounds.” This process continued for 11 groupings of Cs and 4 groupings of Vs. Mastery of oral awareness was indicated when the subject could provide the remaining constituents of each sound grouping when given any of the exemplars (e.g., mouth picture, phoneme, grapheme, verbal label). The subject was in stage one for approximately 4 weeks or 16 treatment hours.

The second stage of the LiPS program, simple nonword training, focused on training phonological awareness of V, CV, and CVC syllables. This stage requires skills that are acquired from the oral awareness stage for the subject to determine the number, order, and sameness/difference of phonemes in speech syllables (markers of phonological awareness). In this stage, the therapist spoke a nonword and asked the subject to repeat the stimulus while looking in the mirror. The subject was asked to sequence the mouth pictures to indicate the phonemes she heard and orally felt in the stimulus. A mirror was used to enhance oral awareness and determine the number and order of phonemes in the nonword. Knowledge of articulatory movements and verbal labels of phonemes are believed to enhance phonological awareness (e.g., parsing a nonword into its constituent phonemes). Next, the nonword was repeated and a new nonword was presented with an addition, omission, or repetition of one phoneme (e.g., "if the old word said feep, show me fip"). The subject said both nonwords, the old and the new, 
aloud and tapped each mouth picture with her finger while saying the phoneme each mouth picture represented in the first nonword. Then, she tapped the same mouth picture again while saying the new nonword. Finally, the subject changed one of the mouth pictures in the sequence of pictures representing the first nonword to represent the new nonword.

Phonological awareness training used a chain of nonsense segments (e.g., /ip/, /ap/, /a/, /pa/, /ap/, /pap/), and exercises began with $\mathrm{V}, \mathrm{CV}$, or $\mathrm{VC}$ and progressed to CVC. After the subject could perform this exercise with 90 to 100 percent accuracy, the mouth pictures were replaced with individual graphemes printed on plastic tiles for reading and spelling exercises. The subject participated in stage two for 58 treatment hours.

\section{Data Analysis}

\section{Visual Inspection}

Three individuals uninvolved in this investigation who were knowledgeable about multiple-baseline singlesubject design studies served as judges for this study. Judges were asked to evaluate by visual inspection the treatment, generalization, and control probe measures (Figure 3). Judges were asked to make their binary judgments by considering ranges of baseline scores as well as the magnitude and slope of changes relative to the timing of conditions. To show treatment or no-treatment effect, we required all three judges to agree.

\section{Time Series Analysis}

To determine magnitude of change in the treatment, generalization, and control probes relative to the timing of conditions, we performed an independent time series analysis of autocorrelated data (ITSACORR) [22] using all baseline points for the pretreatment phase and the final two data points for the posttreatment phase.

Figure 3 displays results for subject performance on treatment (Figure 3(a)), generalization (Figure 3(b)), and control (Figure 3(c)) probes, with the $x$-axis representing session number and the $y$-axis representing accuracy $(0 \%-100 \%)$.

\section{RESULTS}

\section{Research Question 1}

Does phonological treatment improve phonological processing and ability to read nonwords aloud? We meas- ured changes in phonological processing with pre- and posttest assessments using standardized measures (CTOPP and LAC) (Table). We used nonstandardized treatment probes to assess the subject's ability to read aloud VC, CV, and CVC nonwords. Visual inspection of nonword reading aloud data (Figure 3(a)) showed baseline stability and no treatment or maintenance effects. However, ITSACORR analysis showed a marginally significant effect (0.042). In contrast, a second standardized measure of nonword reading (Woodcock Reading Mastery Test-Revised [WRMT-R] Word Attack subtest) showed significant change on pre- to posttreatment assessment (standard score 0 pretreatment and 38 posttreatment).

To summarize the results for the first research question, we found no gains in nonword reading aloud on repeated probes, while a small improvement in phonological processing and nonword reading aloud was evident on standardized pre- and posttreatment assessments.

\section{Research Question 2}

Does treatment generalize to untreated behaviors? A generalization probe of real-word reading aloud of singlesyllable words was administered. Visual inspection of the generalization probe data (Figure 3(b)) showed baseline stability and no treatment or maintenance effects for realword reading aloud. Similarly, ITSACORR analysis for real-word reading aloud showed no effect (0.876).

\section{Research Question 3}

Are treatment and generalization effects maintained following treatment termination? Visual inspection of treatment and generalization data showed no maintenance effects.

\section{Overall Language Function}

The WAB Aphasia Quotient improved from 56.2/100 to 86.5/100. Auditory language comprehension (RTT) improved from 8.817/15 to $10.779 / 15$. Reading comprehension (RCBA performance subtests) improved from 52 (31/60 pretreatment) to 80 percent ( $48 / 60$ posttreatment).

\section{DISCUSSION}

\section{Potential Sources of Observed Improvement in Language Function}

We propose that this subject received two therapies in this study: one planned and less effective, one unplanned and more effective. The planned treatment 

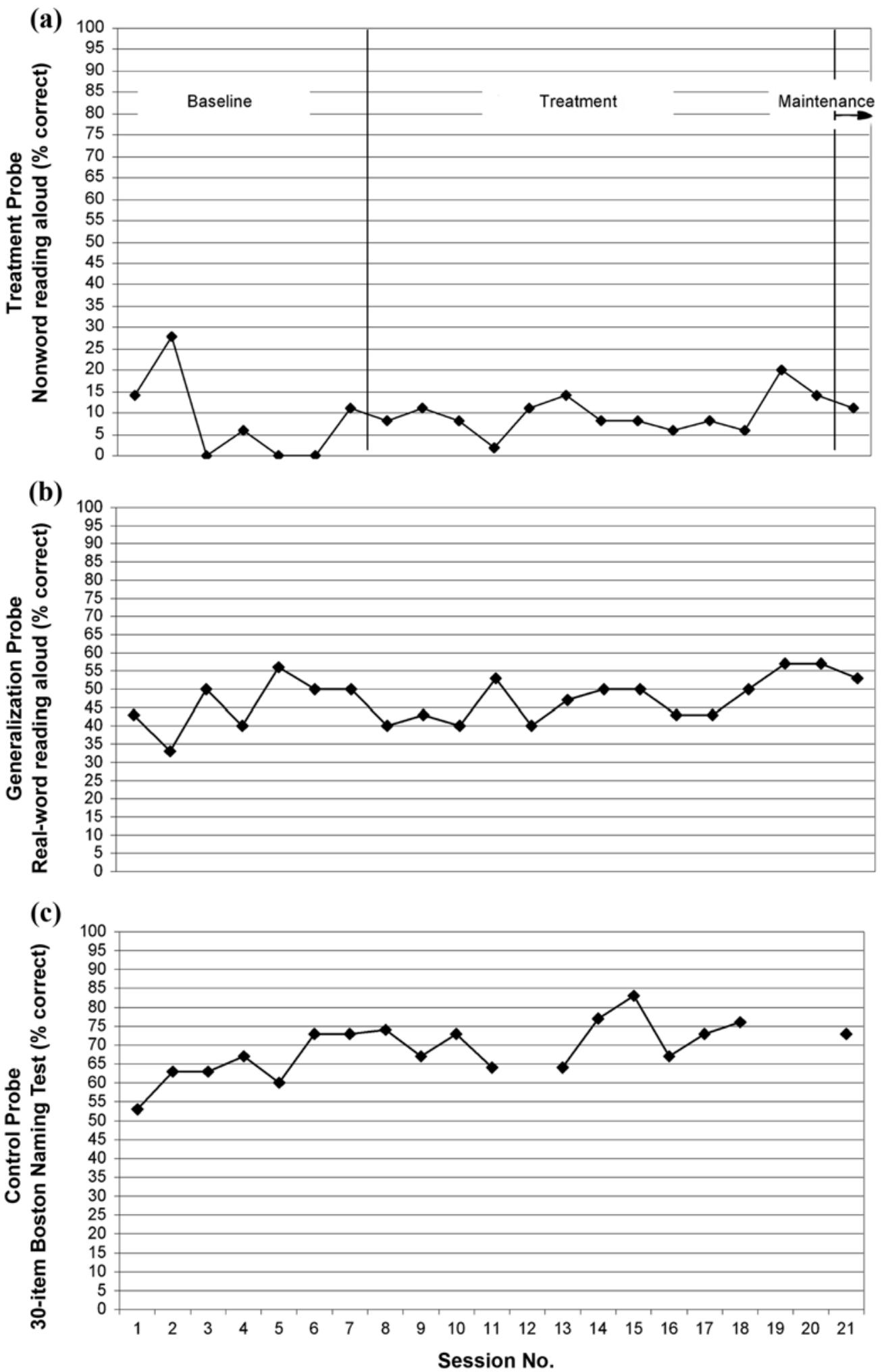

Figure 3.

Baseline, treatment, and maintenance data for percent correct responses on (a) treatment, (b) generalization, and (c) control probes. 
was the intensive phonological therapy and the unplanned treatment was the informal social interaction that occurred extensively in the subject's home. At the time of treatment, the unplanned treatment was viewed merely as incidental informal social interaction and part of the process of being supportive. However, the quantity of this largely verbal interaction and the pattern of changes in outcome measures suggest that it was important and likely the more effective of the two therapies.

During the time that the subject was undergoing phonological treatment, she also participated in intensive and extensive communication with the therapist before daily treatment sessions, on treatment breaks, and following each session. Because therapy would take place inside the subject's home (2-3 days/week), she often showed the therapist family photo albums, prepared lunch (reading recipes), and conversed about daily events. Furthermore, the speech-language pathologist did not aid the subject with phrase-completion cues during conversation; instead she pressed her to communicate on her own. The subject often told stories about family and friends, and the therapist provided little communicative support when the subject experienced anomia (i.e., the therapist did not suggest names of people in the story).

Because this patient simultaneously received these two language therapies, we cannot be certain which therapy produced which improvements in language function. However, the nature of the therapies, the pattern of the results, and the potential for generalization to untreated behaviors of the effects of the two therapies are suggestive.

First, although the experimental phonological treatment produced improvement in standard measures of phonological processing and phonological reading (slight change on CTOP, LAC; modest change on WRMT-R Word Attack subtest), no evidence existed of generalization of the improvement to nonword or real-word reading aloud probes. We conclude that the phonological treatment was less effective in this subject.

Second, the patient did show substantial gains, almost certainly of clinical significance, on standard measures of language function, including the WAB Aphasia Quotient, RTT, and RCBA. That the phonological treatment could have induced these effects when it had no effect on standard phonological processing and reading measures seems unlikely. We conclude that more likely, the unplanned treatment was largely responsible for these gains.

Third, the improvement in phonological processing ability may have worked in concert with the stimulation of language from the intensive conversational framework and resulted in an increase in comprehension and production of language. In the model (Figure 4), the potential value of treating phonological sequence knowledge is that it may enable the use of existing connections between concepts and articulatory motor representations that enable spoken language output, connections that instantiate lexical-semantic knowledge. However, even successful phonological sequence knowledge training may not enable internally generated spoken language without explicit training of lexical-semantic knowledge; in the present case, even very modestly enhanced phonological sequence knowledge, combined with intense language use that strengthened lexical-semantic knowledge, may not have facilitated significantly enhanced speaking ability. Furthermore, either or both treatments could have caused an improvement in speech-motor programming abilities (e.g., apraxia of speech) as indicated by improvement on the repetition and fluency subtests of the WAB.

The unplanned treatment could be viewed as a lexicalsemantic whole-word treatment of oral language production, comprehension, and real-word reading. However, because no systematic relationship exists between word sound or appearance and word meaning, the potential for generalization of lexical-semantic treatments is very modest and limited to the extent that a similarity of word meaning exists and the extent that the lesion affects semantic over orthographic, acoustic, or articulatory representations

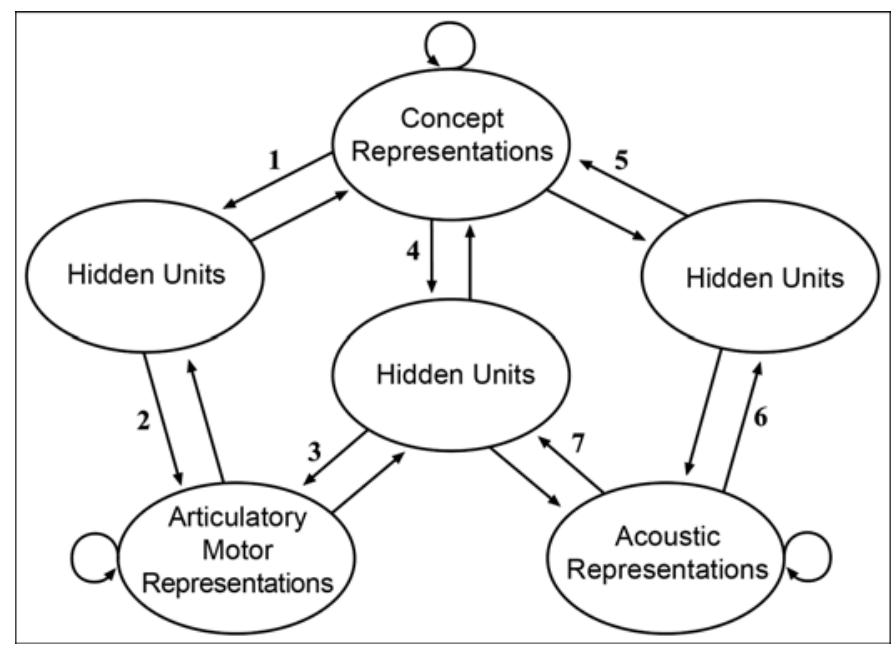

Figure 4.

Connectionist model of phonological processing. Source: Nadeau SE. Phonology: A review and proposals from a connectionist perspective. Brain Lang. 2001;79(3):511-79. [PMID: 11781057] 
[23]. Contrast this with the potential for generalization to all words if the full repertoire of phoneme articulations and phonological sequences could be trained or the generalization to all words (but not other syntactic forms) that occurs when selected grammatical manipulations are trained [24]. Thus, that the lexical-semantic component of the unplanned treatment accounts for the improvement in the WAB Aphasia Quotient, RTT, and RCBA seems unlikely, particularly because the extensive subject-therapist interaction never involved specific study of items in these measures. How then could this inadvertent treatment lead to the demonstrated improvements on these measures? We suggest three possibilities.

First, because of the pressure the therapist placed on the patient to speak during their extensive nonphonological treatment interactions, this treatment could be viewed as a form of constraint-induced language therapy (CILT) [14-15]. CILT can improve language function in at least two ways: (1) in analogy to constraint-induced movement therapy, CILT can potentially overcome an acquired tendency to rely on other modes of communication, such as gesture, in lieu of attempting to engage the impaired linguistic capacity (learned nonuse) [25] and (2) CILT can, by forcing the patient to speak as much as possible, provide the basis for both intensive and extensive experience in multiple aspects of language function. Our patient was relatively isolated socially and over the many years since her stroke, in the absence of significant language therapy, had learned to rely on other modes of communication. She thus constitutes a plausible case of learned nonuse. To the extent that the unplanned treatment overcame this general disposition, we might expect the results to generalize widely, as limited prior studies suggest. That the actual experience of language processing outside the planned phonological treatment led to sufficient improvement in lexical-semantic function to account for the change in WAB, RTT, and RCBA scores seems less likely, since little overlap existed between the semantic content of the conversations and the content of these tests; however, our failure to employ dependent measures to assess these effects precludes any strong conclusions about the extent of generalization.

The second possibility is that the unplanned treatment affected a more fundamental process that underlies language function; for example, the ability to endogenously generate concept representations. That is, a significant component of our patient's linguistic impairment could be characterized as adynamic aphasia [26], and extensive practice in generating concept representations in the course of conversations with the therapist could improve this crucial prelinguistic process.

Finally, the entire course of intensive therapy may have led to an alteration in the patient's general disposition to others, such that she assumed a more active role that demanded greater effort and practice in verbal communication. Blonder has shown that aphasia may lead to a fundamental alteration in patients' positions in their social milieu [27]. Even if formerly in a position of familial and social leadership that demanded extensive verbal communication, a patient with aphasia may be relegated to a more passive role in which less verbal communication may suffice and less social pressure exists to communicate verbally. In this situation, any therapy that succeeds in increasing a patient's confidence in social capacity and communicative ability may lead to greater efforts at verbal communication, which, in turn, may provide the basis for what essentially is a continuation of language therapy outside the speech therapy context and after the completion of formal speech therapy.

\section{Potential Treatability of Components of Language Impairment in Aphasia}

Knowledge of the attributes of aphasic disability that are most predictive of the success or failure of language therapies in general or phonological treatment in particular is of considerable practical and scientific interest. Unfortunately, very little is known about these attributes. Therefore, the discussion that follows of the potential reasons for failure of phonological therapy in our patient must be viewed as a proposal of hypotheses rather than a review of evidence.

Our inquiry into the potential reasons for phonological treatment failure begins with a consideration of the mechanism of phonology as we understand it in terms of a parallel distributed processing (PDP) (connectionist) model. Because the core of that model (Figure 4) has been discussed at length elsewhere [28], we will limit ourselves here to a brief summary. Our model employs the same topography as the Wernicke-Lichtheim information processing model [29], but it also specifies how representations are generated in the modular domains and how knowledge is represented in the links between these domains. Though not tested through simulations, this model is neurally plausible and provides a cogent explanation for a broad range of psycholinguistic phenomena. More generally, connectionist concepts are now deeply 
embedded in and receive enormous support from mainstream neuroscientific research [30-31].

The model posits that the acoustic domain (akin to Wernicke's area) contains large numbers of units located in auditory association cortices that represent acoustic features of phonemes. The articulatory domain (analogous to Broca's area) contains units located predominantly in the dominant frontal operculum that represent discrete articulatory features of speech (as opposed to continuously variable motor programs). The semantic or conceptual domain contains an array of units distributed throughout unimodal, polymodal, and supramodal association cortices that represent semantic features of concepts. Within any domain, a representation corresponds to a specific pattern of activity of all the units, hence the term distributed representation. Each unit within each of these domains is connected via interposed hidden units to many, if not most, of the units in the other domains. During language learning, the strengths of the connections between the units are gradually adjusted so that a pattern of activity involving the units in one domain elicits the correct pattern of activity in the units of a connected domain. The entire set of connections between any two domains forms a pattern-associator network. The hidden unit regions, in conjunction with nonlinear unit properties, enable the association of representations in two connected domains that are arbitrarily related to one another (e.g., word sound and word meaning).

In PDP models, knowledge is stored as patterns of connectivity not only within domains but also between domains. For example, understanding the meaning of a word that is heard is achieved through the connections between the domain that contains the sound features of language and the domain that contains concept features (the acoustic to concepts representations pattern associator, Figure 4, pathway 6-5). This pattern-associator network corresponds to the cognitive neuropsychological concept of a phonological input lexicon [32].

The knowledge that allows a person to translate heard sound sequences into articulatory sequences, and thereby mediates repetition of both real words and nonwords, is contained in the network that connects the acoustic domain to the articulatory motor domain (the acoustic to articulatory motor pattern associator, Figure 4, pathway 7-3). Because this network has acquired, through experience, knowledge of the systematic relationships between acoustic sequences and articulatory sequences, it has learned the sound sequence regularities of the language: the phonemic sequences of joint phonemes, rhymes, syllables, affixes, morphemes, and words characteristic of the language [28].

The knowledge that enables a person to translate a concept into a spoken word (the phonological output lexicon [32]) is contained in two different pattern-associator networks that connect the concept representations domain to the articulatory motor domain (Figure 4, pathways 1-2 and 4-3). These two pattern-associator networks support different forms of knowledge. The indirect concept representations to articulatory motor pathway (Figure 4, pathway 4-3) provides a robust basis for knowledge of sequences and sublexical entities because of the sequence knowledge stored in the acoustic to articulatory motor pattern associator. However, the direct concept representations to articulatory motor pattern associator (Figure 4, pathway 1-2) does not contain much knowledge of sequences and sublexical entities because it translates spatially distributed patterns of activity that correspond to concepts into temporally distributed sequences of activity that correspond to articulated words. This spatial-temporal translation precludes significant acquisition of sequence knowledge and makes this a fundamentally whole-word pathway. The existence of this direct whole-word naming route finds support in studies of subjects with repetition conduction aphasia, some of whom appear to have lost all phonological sequence knowledge (Figure 4, pathway 3/4-7) and do not produce phonological paraphasic errors. They also cannot repeat nonwords but can repeat real words, albeit at times with semantic paraphasic errors [33]. However, a model in which the only link from the concept representations domain to the articulatory motor domain is a direct link (Figure 4, pathway 1-2) cannot account for observations that normal subjects exhibit phonological slips-of-the-tongue and aphasic subjects produce phonemic paraphasias in naming and spontaneous language, which are quite comparable with those produced during repetition. To explain these observations, one must posit access from concept representations to phonological sequence knowledge, as indicated in pathway 4 to 3 of the model (Figure 4).

Finally, to understand how this model is relevant to reading, one must postulate an extension that incorporates pattern-associator networks that support reading via whole-word and phonological routes (Figure 5). This larger model reveals three ways by which phonological treatment might promote the reacquisition of reading via the phonological route after brain damage. Reading via 
the phonological route requires knowledge of the systematic relationships between sequences of graphemes and phonemes. For subjects to learn such sequences, phonemes must be defined as discrete entities consciously accessible from concept representations, a facility that may constitute the basis for phonological awareness. The first phase of phonological treatment, in which subjects learn to conceptualize discrete phonemes in terms of somatosensory and motor configurations of the oropharyngeal, laryngeal, and respiratory apparatus, presumably trains this facility. The acquisition of sequence knowledge by the phonological reading route will be facilitated by the presence of sequence knowledge in the acoustic to articulatory motor pattern associator, as has been shown in simulations by Harm and Seidenberg [34]. The second phase of phonological treatment explicitly trains this acoustic to articulatory sequence knowledge. Although reading via the phonological route benefits from sequence knowledge in the acoustic to articulatory pattern associator, it presumably requires further training of sequence knowledge in the orthographic to articulatory motor pattern associator, the focus of the third phase of phonological treatment.

We are now prepared to address the question that began this section: why was phonological treatment less effective with this subject? Plaut has shown that when a neural network is trained to criterion performance, damaged, and then "rehabilitated" (retrained), the network learns much faster during the retraining phase than it did

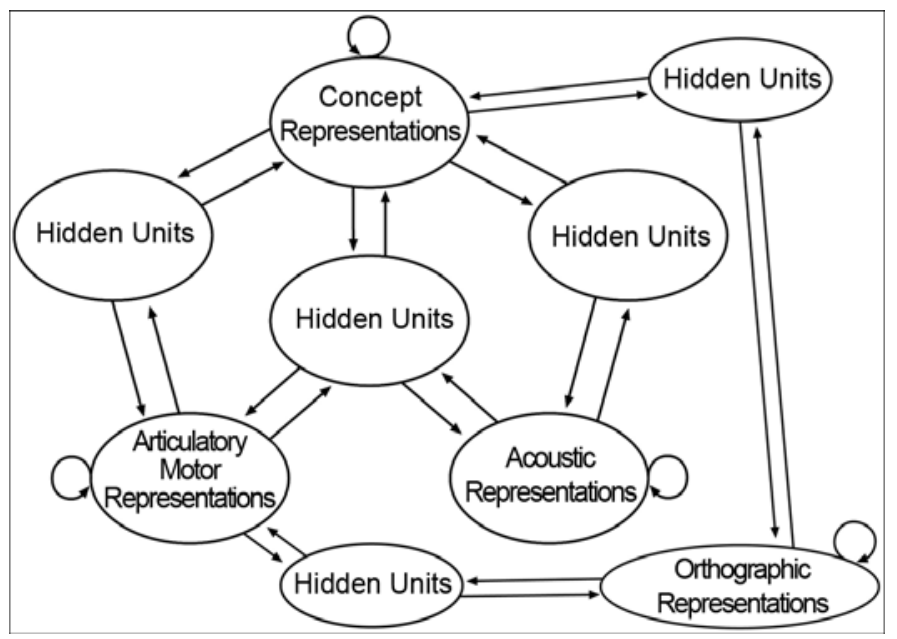

Figure 5.

Connectionist model modified to incorporate pattern-associator networks that support reading. during the original training phase, reflecting the fact that because representations are distributed, knowledge remains in the network even after focal damage [23]. However, the benefits of prior knowledge decline as either the extent of network damage increases, or when, as in the present case of a right-handed person with a very large left-hemisphere lesion, little prior knowledge exists in the unaffected networks of the right hemisphere. In contrast, in a previously reported successful use of a form of phonological therapy involving a subject with acquired phonological alexia [13], the subject was left-handed and the dominant Broca's area was substantially preserved; functional imaging studies demonstrated that following treatment, greater engagement of both the right perisylvian regions and the left Broca's area was exhibited [35].

In infancy and early childhood, the greater plasticity of the brain, coupled with vast exposure to linguistic experience, evidently serves to overcome the problem of lack of existing knowledge. However, in adults with aphasia, the combination of lesser brain plasticity and practical limits to the amount of speech therapy that can be provided likely defines limits to the gains from any form of speech therapy. These limits might be expanded by improving the efficiency of speech therapy, increasing duration of therapy, or developing means for continuation of therapy by patient and family after the formal treatment experience. At the beginning of therapy, our patient demonstrated some rudimentary phonological sequence knowledge by her ability to repeat and read some nonwords and by the fact that confrontation naming was sometimes improved by phonemic cuing. However, this sequence knowledge was apparently not sufficient to provide the basis for acquisition of a useful phonological sequence repertoire because even very extensive phonological treatment succeeded in producing only modest gains on standard measures of phonemic awareness (e.g., LAC) and minimal improvement in phonological production skills at the 3-, 4-, or 5-phoneme syllable level (CTOPP, LAC, WRMT-R Word Attack subtest), i.e., skills dependent on the acquisition of phonological sequence knowledge. During treatment sessions, she ultimately achieved close to 100 percent accuracy in production of individual phonemes but never acquired any phonological sequence knowledge (ability to reproduce trained CV, VC, and CVC syllables). In contrast, several potential mechanisms existed by which forced-use language might have produced clinically significant gains in other measures of language function. 
Determining the minimum existing knowledge in any language domain (phonological sequences included) needed for successful speech-language therapy constitutes one of the major scientific challenges to speechlanguage rehabilitation science. In the present case, we have argued that an adequate pretherapy level of phonological sequence knowledge probably did not exist. Defining an adequate level will require extensive empirical investigation. The adequacy of any given level of knowledge is, furthermore, likely influenced by concurrent dysfunction in other language or even nonlanguage domains (e.g., prefrontal), the motivation of the patient, the degree to which the patient's home environment promotes spoken language use, the efficiency and the duration of therapy, and perhaps other, as yet undefined, factors.

\section{Treatability in Relation to Time Elapsed Since Stroke}

This study provides compelling evidence that even elderly patients may benefit substantially from speech therapy many years or even decades after brain injury. This finding is consistent with the concept that speech therapy conducted long after stroke can engage normal learning mechanisms, which may remain substantially intact despite a focal brain lesion.

\section{CONCLUSIONS}

Notwithstanding the problems posed by potential confounding of the effects of two treatments conducted concurrently (one planned, one inadvertent), the results of this study suggest that (1) phonological therapies are not likely to be successful if a minimal initial level of phonological sequence knowledge does not exist and (2) intensive therapies that strongly pressure subjects to communicate verbally can achieve clinically important gains in communicative ability that generalize far beyond the linguistic material actually used in these therapies. This study demonstrates the importance of a careful analysis of the patient's language ability before choosing a therapeutic strategy. Further research will be needed to fully define predictors of response to specific language therapies and characterize the mechanisms by which forced-use language therapies exert their effects.

One weakness of this study is the internal validity of the single-subject design. In a typical single-subject design, control is demonstrated through replication of the treatment effect a second time or across multiple training sets; however in this study, only one treatment phase involving an omnibus training set incorporating all sounds and sequences was performed. A replication of this study is warranted in which sounds and sequences are separated into subsets for sequential training, rather than presented all at once. Another weakness lies in the administration of the WAB fluency subtest. The pretreatment WAB was administered by a therapist unrelated to the study, while the posttreatment WAB was administered by the treating therapist. The observed change (from 2 to 9) could be because of unreliability of scoring between the two therapists and/or subject ease with the treating therapist during the posttesting session rather than true change in fluency. Finally, additional research is warranted to formally explore the effects of intensive phonological versus forced-used language treatments in a well-controlled group study.

\section{ACKNOWLEDGMENTS}

We would like to thank the subject and her husband for participation in this study. We would also like to thank John Richardson for his assistance with the figures.

Dr. Diane Kendall is now with the Department of Communication Sciences and Disorders, University of Florida, Gainesville, Florida.

This material was based on work supported by the VA RR\&D Brain Rehabilitation Research Center, a VA RR\&D Associate Investigator Award (grant C2438H), the National Institutes of Health (grant P50-DC03888), and a VA RR\&D Career Research Development Award (grant C2743V).

The authors have declared that no competing interests exist.

\section{REFERENCES}

1. Lundberg I, Frost J, Patterson O. Effects of an extensive program for stimulating phonological awareness in preschool children. Read Res Quart. 1988;23(3):263-84.

2. Ball EW, Blachman BA. Phoneme segmentation training: Effect on reading readiness. Ann Dyslexia. 1988;38:208-25.

3. Torgesen JK, Morgan ST, Davis C. The effects of two types of phonological awareness training on word learning in kindergarten children. J Educ Psychol. 1992;84(3):364-70.

4. Alexander AW, Andersen H, Heilman PC, Voeller KS, Torgesen JK. Phonological awareness training and remediation 
of analytic decoding deficits in a group of severe dyslexics. Ann Dyslexia. 1991;41:193-206.

5. De Partz MP. Re-education of a deep dyslexic patient: Rationale of the method and results. Cog Neuropsychol. 1986;3(2):149-77.

6. Matthews C. Serial processing and the "phonetic route:" Lessons learned in the functional reorganization of deep dyslexia. J Commun Disord. 1991;24(1):21-39. [PMID: 2050839]

7. Mitchum CC, Berndt RS. Diagnosis and treatment of the non-lexical route in acquired dyslexia: An illustration of the cognitive neuropsychological approach. J Neurolinguistics. 1991;6(2):103-37.

8. Kendall DL, McNeil MR, Small SL. Rule-based treatment for acquired phonological dyslexia. Aphasiology. 1998; 12(7/8):587-600.

9. Friedman RB, Sample DM, Lott SN. The role of level of representation in the use of paired associate learning for rehabilitation of alexia. Neuropsychologia. 2002;40(2): 223-34. [PMID: 11640944]

10. Friedman RB, Lott SN. Phonologic treatment for deep dyslexia using bigraphs instead of graphemes. Brain Lang. 1996;55:116-18.

11. Lindamood PC, Lindamood PD. The Lindamood Phoneme Sequencing Program for Reading, Spelling, and Speech. Austin (TX): PRO-ED Inc; 1998.

12. Kendall DL, Conway TW, Rosenbek J, Gonzalez Rothi LJ. Phonological rehabilitation of acquired phonologic alexia. Aphasiology. 2003;17(11):1073-96.

13. Conway TW, Heilman PC, Rothi LG, Alexander AW, Adair J, Crosson BA, Heilman KM. Treatment of a case of phonological alexia with agraphia using the Auditory Discrimination in Depth (ADD) program. J Int Neuropsychol Soc. 1998;4(6):608-20. [PMID: 10050366]

14. Maher LM, Kendall DL, Swearengin JA, Pingle K, Holland A, Gonzalez Rothi LJ. Constraint induced language therapy for chronic aphasia: Preliminary findings [abstract]. J Int Neuropsychol Soc. 2003;9(2):192.

15. Pulvermuller F, Neininger B, Elbert T, Mohr B, Rockstroh B, Koebbel P, Taub E. Constraint-induced therapy of chronic aphasia after stroke. Stroke. 2001;32(7):1621-26. [PMID: 11441210]

16. Wagner R, Torgesen J, Rashotte C. The Comprehensive Test of Phonological Processing. Austin (TX): PRO-ED Inc; 1998.

17. Kaplan E, Goodglass H, Weintraub S. The Boston Naming Test. Philadelphia (PA): Lea \& Febiger; 1983.

18. Lindamood PC, Lindamood PD. Lindamood Auditory Conceptualization Test. Austin (TX): PRO-ED Inc; 1979.

19. Kertesz A. The Western Aphasia Battery. Austin (TX): PRO-ED Inc; 1982.
20. McNeil MM, Prescott TE. Revised Token Test. Austin (TX): PRO-ED Inc; 1978.

21. LaPointe LL, Horner J. Reading Comprehension Battery for Aphasia. Austin (TX): PRO-ED Inc; 1979.

22. Crosbie J. Interrupted time-series analysis with brief singlesubject data. J Consult Clin Psychol. 1993;61(6):966-74. [PMID: 8113497]

23. Plaut DC. Relearning after damage in connectionist networks: Toward a theory of rehabilitation. Brain Lang. 1996; 52(1):25-82. [PMID: 8741976]

24. Thompson CK. (2001) Treatment of underlying forms: A linguistic specific approach for sentence production deficits in agrammatic aphasia. In: Chapey R, editor. Language intervention strategies in aphasia and related neurogenic communication disorders. 4th ed. Philadelphia (PA): Lippincott Williams \& Wilkins; 2001. p. 605-25.

25. Taub E, Uswatte G, Pidikiti R. Constraint-induced movement therapy: A new family of techniques with broad application to physical rehabilitation-A clinical review. J Rehabil Res Dev. 1999;36(3):237-51. [PMID: 10659807]

26. Gold M, Nadeau SE, Jacobs DH, Adair JC, Rothi LJ, Heilman KM. Adynamic aphasia: A transcortical motor aphasia with defective semantic strategy formation. Brain Lang. 1997;57(3):374-93. [PMID: 9126422]

27. Blonder LX. Language use. In: Nadeau SE, Gonzalez Rothi LJ, Crosson BA, editors. Aphasia and language: Theory to practice. New York (NY): Guilford Press; 2000. p. 284-95.

28. Nadeau SE. Phonology: A review and proposals from a connectionist perspective. Brain Lang. 2001;79(3):511-79. [PMID: 11781057]

29. Lichtheim L. On aphasia. Brain. 1885;7(4):433-84.

30. Rolls ET, Treves A. Neural networks and brain function. New York (NY): Oxford University Press; 1998. p. 1-418.

31. Rolls ET, Deco G. Computational neuroscience of vision. New York (NY): Oxford University Press; 2002. p. 1-592.

32. Ellis AW, Young AW. Human cognitive neuropsychology. Hillsdale (NJ): Lawrence Erlbaum Associates; 1988.

33. Warrington EK, Shallice T. The selective impairment of auditory verbal short-term memory. Brain. 1969;92(4): 885-96. [PMID: 5364015]

34. Harm MW, Seidenberg MS. Phonology, reading acquisition, and dyslexia: Insights from connectionist models. Psychol Rev. 1999;106(3):491-528. [PMID: 10467896]

35. Adair JC, Nadeau SE, Conway TW, Gonzalez Rothi LJ, Heilman PC, Green IA, Heilman KM. Alterations in the functional anatomy of reading induced by rehabilitation of an alexic patient. Neuropsychiatry Neuropsychol Behav Neurol. 2000;13(4):303-311. [PMID: 11186167]

Submitted for publication January 14, 2005. Accepted in revised form October 5, 2005. 